\title{
Drug therapy in headache
}

\author{
Author: Mark W Weatherall ${ }^{\mathrm{A}}$
}

\begin{abstract}
All physicians will encounter patients with headaches. Primary headache disorders are common, and often disabling. This paper reviews the principles of drug therapy in headache in adults, focusing on the three commonest disorders presenting in both primary and secondary care: tension-type headache, migraine and cluster headache. The clinical evidence on the basis of which choices can be made between the currently available drug therapies for acute and preventive treatment of these disorders is presented, and information given on the options available for the emergency parenteral treatment of refractory migraine attacks and cluster headache.
\end{abstract}

KEYWORDS: Migraine, tension-type headache, cluster headache, analgesics, triptans, CGRP, prophylaxis

\section{Introduction}

Headache is the most common neurological problem encountered by physicians: it accounts for $4.4 \%$ of all consultations in general practice, ${ }^{1}$ approximately $5 \%$ of all medical admissions to hospital, ${ }^{2}$ and approximately $20 \%$ of neurology outpatient consultations. ${ }^{3}$ While tension-type headache (TTH) is the commonest of the primary headache disorders, with a lifetime prevalence of $>90 \%$, it is rarely disabling. Migraine, which affects over $20 \%$ of people at some point in their lives, is typically so debilitating that the World Health Organisation (WHO) equates the disability caused by a day of severe migraine to quadriplegia. Migraine is so common that, even though for many people it is no more than an inconvenience, the cumulative burden of the disorder caused it to rank in the top 40 conditions causing worldwide disability according to the WHO 2012 global burden of disease figures, above all other neurological disorders other than stroke, meningitis and epilepsy; in the UK it ranks third behind stroke and the dementias, causing the loss of 230,000 disabilityadjusted life years annually. ${ }^{4}$ Some of the rarer primary headache disorders, such as cluster headache, paroxysmal hemicrania and short-lasting unilateral neuralgiform headache with conjunctival injection and tearing (SUNCT) syndrome, are among the most painful conditions known, and impose substantial disability on those unfortunate enough to suffer

Author: ${ }^{\text {A }}$ consultant neurologist, Princess Margaret Migraine Clinic, Charing Cross Hospital, London, UK from them. This paper outlines the options available for drug therapy in adults in the three commonest primary headache disorders: TTH, migraine and cluster headache.

\section{Principles of drug therapy in headache}

Drug therapy is only part of an effective approach to the management of headaches. In many cases headache triggers can be identified and lifestyle changes instituted that reduce the frequency of attacks. Rest, sleep and adequate hydration are often important components of successful management regimes. Many headaches, particularly TTHs, will settle spontaneously without the need to therapy. However, if this is not the case, drug therapy is the mainstay of headache management and there are a number of principles that guide the choice and deployment of effective medication.

First and foremost it is important to make the correct diagnosis. The International Classification of Headache Disorders (ICHD) comprises a large number of primary and secondary headache disorders, ${ }^{5}$ but in everyday practice the commonest conditions that will present to physicians are migraine, TTH and cluster headache. This review will concentrate on the treatment of these three conditions. The ICHD criteria for these three conditions are given in Box 1; it is important to remember that not everyone's headache has 'read the textbook', and that it is permissible and necessary to make a working diagnosis even if the history does not include all the features that can be seen in any given condition.

Establishing a diagnosis then allows the appropriate drugs to be chosen. While some acute and preventive medications have a broad spectrum of effect over a number of conditions, others are specific to particular types of headache. These drugs must be given at effective doses, using the most appropriate route of delivery for the patient. Acute treatments should be given early when the pain is mild, ${ }^{6}$ with consideration given to using combinations of drugs to treat pain and other associated symptoms such as nausea. Patient comorbidity and prior experience will guide treatment choices in some cases. Finally, when considering acute treatment, it is important to avoid drugs with high potential for medication overuse headache, and advise patients on appropriate limits with regard to frequency of administration.

Preventive treatment aims to reduce the frequency, severity and duration of headaches, and avoid medication-overuse headache. There are no hard and fast rules on when preventive treatment should be offered, but typically one would discuss this with patients when there are attacks causing frequent 
Box 1. ICHD-3 definitions: TTH, migraine (without aura) and cluster headache.

\section{Infrequent TTH}

A. At least 10 episodes of headache occurring on $<1$ day/month ( $<12$ days/year) and fulfilling criteria B-D.

B. Lasting from 30 minutes to 7 days.

C. $\geq 2$ of the following four characteristics:

$>$ bilateral location

> pressing or tightening (non-pulsating) quality

$>$ mild or moderate intensity

$>$ not aggravated by routine physical activity.

D. Both of the following:

$>$ no nausea or vomiting

$>$ no more than one of photophobia or phonophobia.

E. Not better accounted for by another ICHD-3 diagnosis.

\section{Migraine without aura}

A. At least five attacks fulfilling criteria B-D.

B. Headache attacks lasting 4-72 hours (untreated or unsuccessfully treated).

C. Headache has $\geq 2$ of the following characteristics:

$>$ unilateral location

$>$ pulsating quality

$>$ moderate or severe pain intensity

> aggravation by or causing avoidance of routine physical activity (eg walking, climbing stairs).

D. During headache $\geq 1$ of the following:

$>$ nausea and/or vomiting

> photophobia and phonophobia.

E. Not better accounted for by another ICHD-3 diagnosis.

\section{Cluster headache}

A. At least five attacks fulfilling criteria B-D.

B. Severe or very severe unilateral orbital, supraorbital and/or temporal pain lasting 15-180 minutes (when untreated).

C. Either or both of the following:

$>\geq 1$ of the following ipsilateral symptoms or signs:

> conjunctival injection and/or lacrimation

$>$ nasal congestion and/or rhinorrhoea

$>$ eyelid oedema

$>$ forehead and facial sweating

$>$ forehead and facial flushing

$>$ sensation of fullness in the ear

$>$ miosis and/or ptosis

$>$ a sense of restlessness or agitation.

D. Frequency from $1 / 2$ day to $8 /$ day for more than half the time when active.

E. Not better accounted for by another ICHD-3 diagnosis.

ICHD-3 = International Classification of Headache Disorders-3; TTH = tensiontype headache. disability (eg $\geq 2$ attacks/month that produce disability lasting for 3 days or more), where acute treatments are either contraindicated or ineffective, or where the headache phenotype is unusual, such as hemiplegic migraine, migraine with prolonged aura or cluster headache. Before prescribing, the benefits (reduction in attacks) and risks (adverse effects) of treatment should be discussed, and patients reminded that acute treatment will still be required.

\section{Tension-type headache}

TTH is by definition mild or moderate at worst, and often needs no specific therapy unless attacks are intrusive or particularly frequent. Guidelines for the treatment of TTH have been published by the British Association for the Study of Headache (BASH) and the European Federation of Neurological Societies (EFNS). ${ }^{7,8}$ Protocols have recently been published in the Cochrane library for forthcoming reviews of several acute and preventive treatments for TTH.

\section{Acute treatment of TTH}

Paracetamol 1,000 mg, aspirin 500-1,000 mg and several nonsteroidal anti-inflammatories (NSAIDs), including ibuprofen 200-800 mg, ketoprofen $25 \mathrm{mg}$ and naproxen 250-500 mg, have been shown to be effective in the acute treatment of TTH. ${ }^{9-12}$ Paracetamol has not been shown to be effective at doses below $1,000 \mathrm{mg}$ and some studies have shown it to be less effective than NSAIDs. Combination analgesics containing caffeine may be more effective still, ${ }^{13}$ but are likely to carry a greater propensity to induce medication overuse headache - though it is unlikely that patients with TTH alone, without a personal or family history of migraine, are susceptible to this condition. ${ }^{14}$

\section{Preventive treatment of TTH}

There are few good-quality trials of preventive treatment of TTH. The best evidence exists for amitriptyline $75 \mathrm{mg}$ daily, the first reports of this dating back over 50 years. ${ }^{15,16}$ This medication is best commenced at $10-25 \mathrm{mg}$ daily, and titrated steadily upwards until a positive response is achieved or intolerable side effects (typically drowsiness, dry eyes, dry mouth or constipation) are experienced. Mirtazipine $30 \mathrm{mg}$ daily and venlafaxine $150 \mathrm{mg}$ daily, modern antidepressants that affect both serotonin and noradrenaline metabolism, have also been shown to be effective in reducing the frequency of TTH, ${ }^{17,18}$ but selective serotonin reuptake inhibitors (SSRIs) have not.

\section{Migraine}

Migraine causes moderate or severe pain, and almost inevitably requires acute treatment. There are no acute treatments currently available that block the physiological processes underlying aura (cortical spreading depression), but a series of analgesics have been shown to reduce migraine pain, and other drugs are available to treat the nausea that is commonly found in migraine attacks. The National Institute for Health and Care Excellence (NICE) have recently published guidance on the diagnosis and treatment of migraine, and further consensus guidelines have been published by BASH, the American Headache Association (AHA) and the European Headache Federation (EHF).,19 


\section{Acute treatment of migraine}

Several Cochrane systematic reviews exist of evidence base for commonly used acute treatments for migraine. Of the commonly available over-the-counter drug therapies, paracetamol 1,000 $\mathrm{mg}$ is superior to placebo (number needed to treat (NNT) 12 for two-hour pain freedom), its effectiveness being significantly increased by the addition of metoclopramide $10 \mathrm{mg} .{ }^{20}$ Aspirin $900-1,000 \mathrm{mg}$ is more effective than paracetamol (NNT 8.1), as is diclofenac $50 \mathrm{mg}$ (NNT 8.9) and ibuprofen $400 \mathrm{mg}$ (NNT 7.2), while naproxen 500-825 mg is not (NNT 11). ${ }^{21-24}$ There is no consensus on which antiemetic is most helpful for migraine-associated nausea and vomiting; in addition to metoclopramide, domperidone $10 \mathrm{mg}$ (higher doses now being regarded as inadvisable because of the risk of QT prolongation), buccal prochlorperazine or cyclizine may be considered. If nausea cannot be controlled, then analgesics taken per rectum can be helpful.

If simple analgesics do not control migraines adequately, patients are often prescribed or procure combination analgesics containing paracetamol and codeine. There are very few trials of opiate medication in migraine; one study indicates that a combination of codeine $25 \mathrm{mg}$ and paracetamol 1,000 mg is no better than aspirin. ${ }^{25}$ Given the known risks of rapid habituation to opiates, and of developing rebound headache and medication overuse, the use of opiates for migraine should be a last resort when all other acute treatments are either ineffective or contraindicated.

Therefore if simple analgesics are ineffective, patients should be offered triptans. Triptans are 5-hydroxytryptamine $(\mathrm{HT})_{1 \mathrm{~B} / 1 \mathrm{D}}$ agonists, believed to exert their effect through modulation of the release of calcitonin gene-related peptide (CGRP) both peripherally and centrally. There are seven different triptans currently available in a number of formulations giving various options for route of delivery, including oral tablets or melts, nasal sprays and subcutaneous injection. Inhaled and transdermal formulations are under development. All the seven currently available triptans sumatriptan, zolmitriptan, rizatriptan, naratriptan, eletriptan, almotriptan and frovatriptan - have been shown to be effective in the acute treatment of migraine, with NNTs between 2.3 and 11.3. Table 1 presents the most recent meta-analyses for each formulation, ${ }^{26-31}$ and Fig 1 summarises the relative efficacy of simple analgesics and the triptans.

If first choice triptan therapy proves ineffective despite being taken early in the attack (that is, as soon as the pain starts), then one should consider increasing the dose, altering the mode of delivery or trying another member of the class. If headache recurrence is a problem, then one can consider using naratriptan or frovatriptan, which have longer half-lives than the other triptans, or using a combination of a triptan and longacting NSAID such as naproxen.

Triptans are generally very safe and well tolerated. The commonest side effects are nausea, and tight or burning sensations in the head, neck or chest; the latter can be disconcerting but are not associated with either myocardial ischaemia or muscle breakdown. Serious cardiovascular adverse effects are very rare, occurring in less than one in one million exposures. ${ }^{32}$ Nonetheless, triptans are contraindicated in patients with uncontrolled hypertension, previous myocardial infarction, TIA or stroke, and peripheral vascular disease.
Table 1. NNTs for pain freedom at two hours with

triptans in acute migraine.

\begin{tabular}{|c|c|c|}
\hline Reference(s) & Triptan & NNT \\
\hline \multirow{2}{*}{$\begin{array}{l}\text { Cochrane reviews: } \\
\text { Lance and Curran }^{15}\end{array}$} & SUM 25 & $6.2(4.9-8.5)$ \\
\hline & SUM 50 & $6.1(5.5-6.9)$ \\
\hline Bendtsen et $a l^{16}$ & SUM 100 & $4.7(4.3-5.1)$ \\
\hline \multirow[t]{7}{*}{ Bendtsen and Jensen ${ }^{17}$} & SUM SC & $2.3(2.1-2.4)$ \\
\hline & $S U M+N A P$ & $4.9(4.3-5.7)$ \\
\hline & SUM NS 10 & $7.3(5.5-11)$ \\
\hline & SUM NS 20 & $4.7(4.0-5.9)$ \\
\hline & ZOM 2.5 & $5.1(4.7-5.7)$ \\
\hline & ZOM 5 & $4.5(4.2-4.9)$ \\
\hline & ZOM 10 & $3.1(2.7-3.7)$ \\
\hline Zissis et $a l^{18}$ & ALMO 12.5 & $5.2(3.5-7.0)$ \\
\hline \multirow[t]{3}{*}{ Loder et al ${ }^{19}$} & RIZ 10 & $3.2(2.9-3.5)$ \\
\hline & NAR 2.5 & $8.2(5.0-21.4)$ \\
\hline & FROVA 2.5 & $11.3(9.3-14.3)$ \\
\hline \multirow[t]{2}{*}{ Derry and Moore ${ }^{20}$} & ELE 80 & $3.7(3.4-4.2)$ \\
\hline & ELE 40 & $4.5(3.9-5.1)$ \\
\hline
\end{tabular}

ALMO = almotriptan; $E L E$ = eletriptan; $F R O V A$ = frovatriptan; $\mathrm{NAP}=$ naproxen; NAR = naratriptan; NNTs = numbers needed to treat; NS = nasal spray; RIZ = rizatriptan; SUM = sumatriptan; ZOM = zolmitriptan.

While not generally recommended for use in pregnancy or breast feeding, both a population-based epidemiological study and a long-term cohort study have concluded that there is no evidence that triptan use causes any increase in the rates of significant foetal abnormality. ${ }^{33,34}$

\section{Refractory migraine}

When standard acute therapies fail, patients may present to physicians in 'status migrainosus', that is, with a persistent migraine refractory to treatment, requiring parenteral therapy. A number of options exist in addition to subcutaneous sumatriptan, already discussed. A recent systematic review by the Canadian Headache Society strongly recommended the use of prochlorperazine ( $10 \mathrm{mg}$ iv), lysine acetylsalicylic acid (1.0-1.8 g iv), metoclopramide (10-20 mg iv) or ketorolac (60 mg im or $30 \mathrm{mg}$ iv). ${ }^{35}$ Weaker evidence exists for the use of chlorpromazine ( $25 \mathrm{mg}$ iv) and dihydroergotamine (1 mg im or sc). If migraine persists despite the options outlined above, repetitive infusions of intravenous dihydroergotamine at $1 \mathrm{mg}$ tds up to a total of $10 \mathrm{mg}$ can be effective. ${ }^{36}$

\section{Preventive treatment of migraine}

The first randomised controlled trial of a preventive treatment for migraine (methysergide) was published in 1964. Over the succeeding five decades, numerous further trials have appeared: guidance on the choices available for the preventive treatment of migraine can be found in the 2012 NICE guidelines (currently under revision), and in the consensus statements of $\mathrm{BASH}$, the AHA and the EHF. 


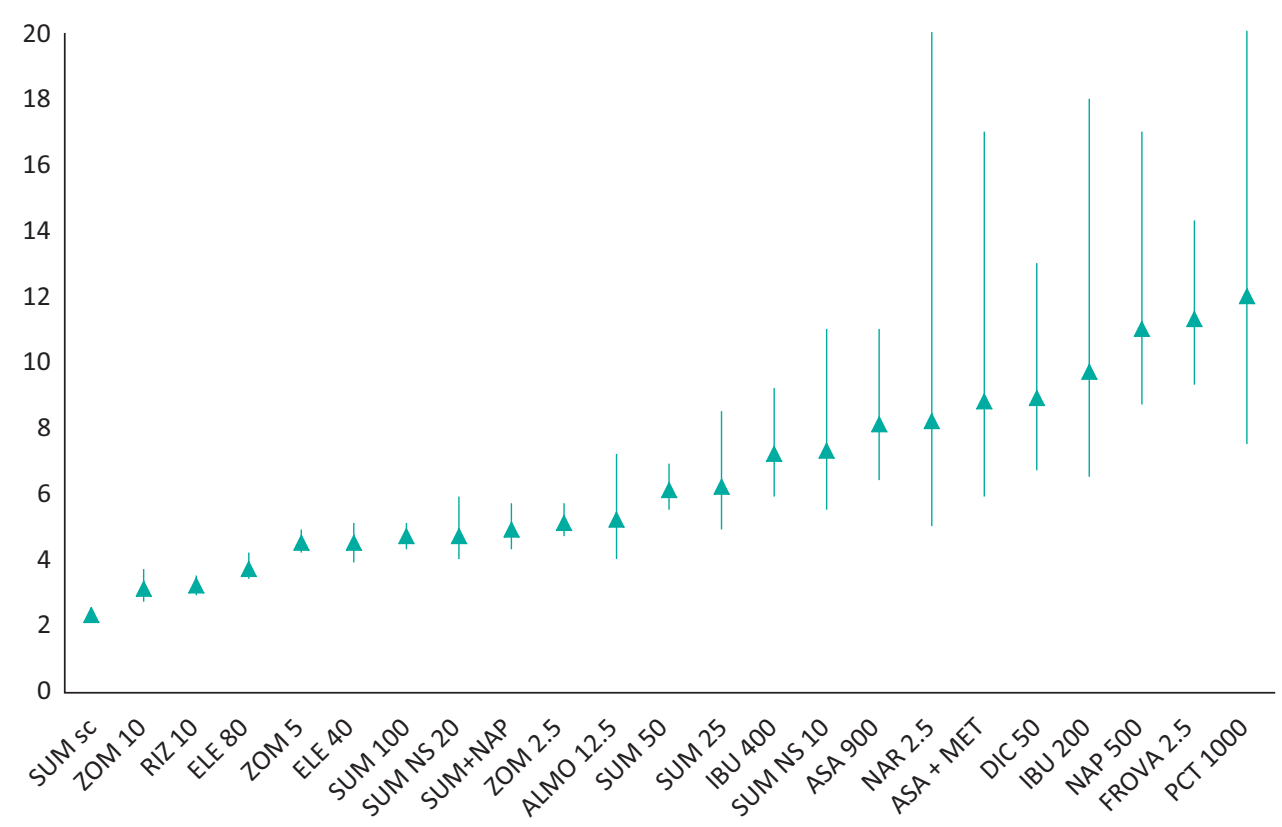

Fig 1. NNTs for two-hour pain freedom for acute migraine. $\mathrm{ALMO}=$ almotriptan; $\mathrm{ASA}=$ aspirin; DIC = diclofenac; $E L E=$ eletriptan; $F R O V A$ = frovatriptan; $\mathrm{IBU}=$ ibuprofen; $\mathrm{MET}=$ metoclopramide; $\mathrm{NAP}=$ naproxen; $\mathrm{NAR}=$ naratriptan; NNTs = numbers needed to treat; $\mathrm{NS}=$ nasal spray; $\mathrm{PCT}=$ paracetamol; RIZ = rizatriptan; $S U M=$ sumatriptan; $\mathrm{ZOM}=$ zolmitriptan.
While NICE has recommended topiramate as a firstline migraine preventive, many physicians regard its side effect profile as a deterrent to its use at that stage. Many headache specialists will use either a $\beta$-blocker or a tricyclic antidepressant in the first instance, depending on patient preference and comorbidity. Pure $\beta$-blockers, such as propranolol, nadolol, metoprolol, atenolol, timolol and bisoprolol, have proven efficacy in migraine prophylaxis, whereas $\beta$-blockers with partial agonist properties do not. Trials comparing one $\beta$-blocker against another generally show equivalent effect with equipotent doses, with overall responder rates (that is, the percentage of patients experiencing a 50\% reduction in headache days) being 53\% compared with $31 \%$ with placebo. ${ }^{37}$ Common side effects include fatigue (often in the form of decreased exercise tolerance), dizziness, insomnia, depression and decreased libido; where CNS side effects are prominent, atenolol can be helpful as it has lower CNS penetrance than the other effective $\beta$-blockers. $\beta$-blockers are contraindicated in patients with asthma, COPD, Raynaud's and peripheral vascular disease.

Amitriptyline was first reported to be helpful for migraine in the late 1960s; in 1979 a small double-blinded placebocontrolled randomised trial of amitriptyline $25 \mathrm{mg}$ showed a responder rate of $55 \%$ versus $34 \%$ with placebo. ${ }^{38}$ Since then a number of comparative trials have demonstrated the non-inferiority of amitriptyline to other standard preventives, including propranolol and topiramate. ${ }^{39,40}$ Side effects, such as fatigue, dry eyes, dry mouth or constipation, are common; anecdotally, other tricyclics, such as nortriptyline or dosulepin, may be better tolerated.

While several anticonvulsants have been shown to be effective for migraine prophylaxis, good-quality evidence only exists for topiramate and sodium valproate. ${ }^{41}$ Negative trials exist for lamotrigine, oxcarbazepine and vigabatrin, and only very poorquality studies exist for carbamazepine and clonazepam. Initial trials suggesting a role for gabapentin in migraine prophylaxis have been largely discredited and the evidence now suggests that this medication is ineffective. ${ }^{42}$ Only small open trials exist for pregabalin and tiagabine; positive but small randomised controlled trials and non-inferiority trials indicate that zonisamide and levetiracetam might be worthy of further study. Topiramate was studied in a series of landmark trials that showed significant responder rates of approximately $50 \%$ versus $23 \%$ with placebo at $100 \mathrm{mg}$ daily, and non-inferiority to propranolol. ${ }^{43}$ Adverse effects are common and often limiting: these include paresthesia, fatigue, tremor, weight loss, visual disturbances (including acute glaucoma), kidney stones and cognitive problems, such as word-finding difficulties, agitation, confusion, depression or psychosis. Trials of sodium valproate as a preventive treatment for migraine date back to the early 1990 s. Pooled results from several trials show a responder rate of 50\% versus $18 \%$ with placebo. ${ }^{44}$ Sodium valproate may cause fatigue, nausea, tremor, weight gain, hair loss, thrombocytopenia, and liver or pancreatic dysfunction. It must also be used with extreme caution in women of child-bearing age as increasing evidence links the use of valproate in pregnancy with adverse neurodevelopmental outcomes in the child. ${ }^{45}$

There are a few other oral migraine preventive therapies with some supportive evidence from clinical trials. Candesartan $16 \mathrm{mg}$ was shown in a small double-blind placebo-controlled crossover trial over 12 weeks to reduce the mean number of headache days from 18.5 with placebo to 13.6 with candesartan, with a $50 \%$ responder rate of $40.4 \% .{ }^{46}$ In a 2013 trial by the same Norwegian group, candesartan was non-inferior to propranolol, the proportion of responders being significantly higher with candesartan (43\%) and propranolol (40\%) than placebo $(23 \%){ }^{47}$ The calcium channel blocker flunarizine is widely used in Europe and South America as a first-line prophylactic medication, and is regarded by many as the drug of choice for severe aura, hemiplegic migraine and migrainous vertigo. One well-conducted trial shows it to be as effective as propranolol. ${ }^{48}$ It is available in the UK on a 'named patient' 
basis. Riboflavin (vitamin B2) showed a responder rate of 56\% versus $19 \%$ on placebo in one small trial, though the baseline attack frequency was very low. ${ }^{49}$

The most recent addition to the options available for the preventive treatment of migraine is Botox (onabotulinum toxin A). Reports of the potential usefulness of this therapy for migraine date back to the mid 1990s, but Botox has only been shown to be unequivocally effective in patients with chronic migraine, that is, headaches on $\geq 15$ days/month, of which at least 8 are migraines or treated as such. Two large high-quality trials (the PREEMPT study) demonstrate a statistically and clinically significant benefit of Botox over placebo. ${ }^{50}$ On this basis, Botox was licensed for chronic migraine and ultimately approved by NICE for use in the NHS in patients unresponsive to $\geq 3$ previous preventive treatments in whom medication overuse headache has been addressed.

\section{Cluster headache}

Cluster headache inevitably requires drug therapy. Approximately $80 \%$ of cluster headache sufferers have the episodic form of the disorder in which attacks come in bouts lasting days to weeks, with significant periods of remission in between. These patients require acute treatments, as well as transitional treatments to try to abort or shorten the bout. The remaining $20 \%$ of sufferers have the chronic form of the disorder, in which acute treatments need to be supplemented with preventive therapy. Useful guidelines for treatment have been published by the EFNS. ${ }^{51}$

\section{Acute and transitional treatments for cluster headache}

Only two acute treatments have been shown to be effective for cluster headaches: triptans and high-flow oxygen. Because of the rapid onset and severity of cluster pain, oral triptans are not effective. Subcutaneous sumatriptan $6 \mathrm{mg}$ has been shown to render $48 \%$ of patients pain free at 15 minutes versus $17 \%$ placebo (NNT 3.3), whereas intranasal zolmitriptan $10 \mathrm{mg}$ only rendered $12 \%$ pain free versus $3 \%$ placebo (NNT 11 ). ${ }^{52}$ Intranasal sumatriptan $20 \mathrm{mg}$ was shown in one trial to render $57 \%$ patients pain free at 30 minutes versus $26 \%$ placebo. ${ }^{53}$ High-flow $100 \%$ oxygen ( $12 \mathrm{l} / \mathrm{min}$ delivered by face mask) has been shown to render $78 \%$ pain free at 15 minutes versus $20 \%$ placebo. ${ }^{54}$ For most patients, a combination of a nonoral triptan and oxygen will be effective; unlike patients with migraine, cluster headache sufferers do not seem to run into problems with medication overuse headache unless they have a personal or strong family history of migraine. ${ }^{55}$

The use of oral steroids to settle a bout of cluster headache dates back to the 1970s. The original observation, that prednisolone at doses $\geq 40 \mathrm{mg}$ daily can reduce or abolish cluster pain in a significant proportion of sufferers, but that recurrence is common (up to $80 \%$ ) as the dose is tailed off, remains true. ${ }^{56}$ Steroids therefore help by aborting the bout in a small proportion of sufferers, providing respite and the chance to introduce another preventive treatment in others.

\section{Preventive treatment for cluster headache}

The usual first-line preventive treatment for cluster headache is verapamil. This has been shown to be effective at a dose of $360 \mathrm{mg}$ daily, ${ }^{57}$ though in some cases patients may benefit from titration of the dose as high as $960 \mathrm{mg}$ daily. Patients may experience dizziness, fatigue and constipation on this medication, and the ECG should be checked regularly (even when established on a stable dose) to look for the development of a prolonged PR interval and ultimately heart block. ${ }^{58}$

If verapamil is not tolerated or effective, then typical secondline therapies include topiramate and lithium carbonate. Only open-label studies exist for the former, and while one crossover study suggested that the latter was as effective as verapamil, ${ }^{59}$ a subsequent placebo-controlled randomised controlled trial of lithium carbonate (modified release) $800 \mathrm{mg}$ daily did not demonstrate superiority over placebo. ${ }^{60}$ Similarly, the positive results seen in open studies of sodium valproate could not be replicated in a controlled trial. ${ }^{61}$ There is one trial that shows that melatonin $10 \mathrm{mg}$ can be effective for the prevention of cluster headache.$^{62}$ Further studies are urgently required in this area.

\section{Conclusion}

Numerous options exist for both acute and preventive drug therapy in TTH, migraine and cluster headache. By choosing options backed by good-quality clinical trial evidence, and using them according to the principles outlined in the first section of this paper, it is possible to make a significant difference to the lives of the vast majority of patients with these primary headache disorders. For those who remain refractory to currently available treatments, the approach of a new generation of drugs targeted at CGRP, the lynchpin molecule of both migraine and cluster headache, holds out hope of improvement in the not-too-distant future. ${ }^{63}$.

\section{References}

1 Tepper S, Dahlöf C, Dowson A et al. Prevalence and diagnosis of migraine in patients consulting their physician with a complaint of headache: data from the Landmark Study. Headache 2004;44:856-64.

2 Weatherall M. Acute neurology in a twenty-first century district general hospital. J R Coll Physicians Edinb 2006;36:196-200.

3 Stone J, Carson A, Duncan R et al. Who is referred to neurology clinics? - the diagnoses made in 3781 new patients. Clin Neurol Neurosurg 2010;112:747-51.

4 World Health Organisation. WHO global health estimates: DALYs, 2000-2012. Geneva: WHO, 2012. Available online at www.who.int/ healthinfo/global_burden_disease/en/ [Accessed 31 January 2015].

5 Headache Classification Committee of the International Headache Society. The International Classification of Headache Disorders, 3rd edition (beta version). Cephalalgia 2013;33:629-808.

6 Goadsby P, Zanchin G, Geraud G et al. Early vs. non-early intervention in acute migraine-'Act when Mild (AwM)'. A double-blind, placebo-controlled trial of almotriptan. Cephalalgia 2008;28:383-91.

7 MacGregor E, Steiner T, Davies P, for the British Association for the Study of Headache. Guidelines for all healthcare professionals in the diagnosis and management of migraine, tension-type, cluster, and medication overuse headache. 3rd edition (1st revision). Available online at www.bash.org.uk/wp-content/uploads/2012/07/10102-BASHGuidelines-update-2_v5-1-indd.pdf [Accessed 31 January 2015].

8 Bendtsen L, Evers $\mathrm{S}$, Linde $\mathrm{M}$ et al. EFNS guideline on the treatment of tension-type headache - report of an EFNS task force. Eur J Neurol 2010;17:1318-25.

9 Prior M, Cooper K, May L et al. Efficacy and safety of acetaminophen and naproxen in the treatment of tension-type headache. A randomized, double-blind, placebo-controlled trial. Cephalalgia 2002;22:740-8. 
10 Schachtel B, Furey S, Thoden W. Nonprescription ibuprofen and acetaminophen in the treatment of tension-type headache. J Clin Pharmacol 1996;36:1120-5.

11 Steiner T, Lange R. Ketoprofen $(25 \mathrm{mg})$ in the symptomatic treatment of episodic tension-type headache: double-blind placebocontrolled comparison with acetaminophen $(1000 \mathrm{mg})$. Cephalalgia 1998;18:38-43.

12 Steiner T, Lange R, Voelker M. Aspirin in episodic tension-type headache: placebo-controlled dose-ranging comparison with paracetamol. Cephalalgia 2003;23:59-66.

13 Migliardi J, Armellino J, Friedman $\mathrm{M}$ et al. Caffeine as an analgesic adjuvant in tension headache. Clin Pharmacol Ther 1994;56:576-86.

14 Bahra A, Walsh M, Menon S et al. Does chronic daily headache arise de novo in association with regular use of analgesics? Headache 2003;43:179-90.

15 Lance J, Curran D. Treatment of chronic tension headache. Lancet 1964;1:1236-9.

16 Bendtsen L, Jensen R, Olesen J. A non-selective (amitriptyline), but not a selective (citalopram), serotonin reuptake inhibitor is effective in the prophylactic treatment of chronic tension-type headache. J Neurol Neurosurg Psychiatry 1996;61:285-90.

17 Bendtsen L, Jensen R. Mirtazapine is effective in the prophylactic treatment of chronic tension-type headache. Neurology 2004;62:1706-11.

18 Zissis N, Harmoussi S, Vlaikidis N et al. A randomized, doubleblind, placebo-controlled study of venlafaxine XR in out-patients with tension-type headache. Cephalalgia 2007;27:315-24.

19 Loder E, Burch R, Rizzoli P. The 2012 AHS/AAN guidelines for prevention of episodic migraine: a summary and comparison with other recent clinical practice guidelines. Headache 2012;52:930-45.

20 Derry S, Moore R. Paracetamol (acetaminophen) with or without an antiemetic for acute migraine headaches in adults. Cochrane Database Syst Rev 2013 Apr 30;4:CD008040.

21 Kirthi V, Derry S, Moore R. Aspirin with or without an antiemetic for acute migraine headaches in adults. Cochrane Database Syst Rev 2013 Apr 30;4:CD008041.

22 Derry S, Rabbie R, Moore R. Diclofenac with or without an antiemetic for acute migraine headaches in adults. Cochrane Database Syst Rev 2013 Apr 30;4:CD008783.

23 Rabbie R, Derry S, Moore R. Ibuprofen with or without an antiemetic for acute migraine headaches in adults. Cochrane Database Syst Rev 2013 Apr 30;4:CD008039.

24 Law S, Derry S, Moore R. Naproxen with or without an antiemetic for acute migraine headaches in adults. Cochrane Database Syst Rev 2013 Oct 20;10:CD009455.

25 Boureau F, Joubert JM, Lasserre V et al. Double-blind comparison of an acetaminophen $400 \mathrm{mg}$-codeine $25 \mathrm{mg}$ combination versus aspirin $1000 \mathrm{mg}$ and placebo in acute migraine attack. Cephalalgia 1994;14:156-61.

26 Derry C, Derry S, Moore R. Sumatriptan (all routes of administration) for acute migraine attacks in adults - overview of Cochrane reviews. Cochrane Database Syst Rev 2014 May 28;5:CD009108.

27 Law S, Derry S, Moore R. Sumatriptan plus naproxen for acute migraine attacks in adults. Cochrane Database Syst Rev 2013b Oct 21;10:CD008541.

28 Bird S, Derry S, Moore R. Zolmitriptan for acute migraine attacks in adults. Cochrane Database Syst Rev 2014 May 21;5:CD008616.

29 Chen L, Ashcroft D. Meta-analysis examining the efficacy and safety of almotriptan in the acute treatment of migraine. Headache 2007;47:1169-77.

30 Adelman J, Belsey J. Meta-analysis of oral triptan therapy for migraine: number needed to treat and relative cost to achieve relief within 2 hours. J Managed Care Pharm 2003;9:45-52.

31 Oldman A, Smith L, McQuay H et al. Pharmacological treatments for acute migraine: quantitative systematic review. Pain 2002;97:247-57.
32 Dodick D, Lipton R, Martin V et al. Consensus statement: cardiovascular safety profile of triptans (5HT agonists) in the acute treatment of migraine. Headache 2004;44:414-25.

33 Nezvalová-Henriksen K, Spigset O, Nordeng H. Triptan safety during pregnancy: a Norwegian population registry study. Eur J Epidemiol 2013;28:759-69.

34 Ephross S, Sinclair S. Final results from the 16-year sumatriptan, naratriptan, and treximet pregnancy registry. Headache 2014;54:1158-72.

35 Orr S, Aubé M, Becker W et al. Canadian Headache Society systematic review and recommendations on the treatment of migraine pain in emergency settings. Cephalalgia 2014; 35:271-284.

36 Nagy A, Gandhi S, Bhola R et al. Intravenous dihydroergotamine for the inpatient management of refractory primary headaches. Neurology 2011;77:1827-32.

37 Linde K, Rossnagel K. Propranolol for migraine prophylaxis. Cochrane Database Syst Rev 2004;(2):CD003225.

38 Couch J, Hassanein R. Amitriptyline in migraine prophylaxis. Arch Neurol 1979;36:695-9.

39 Ziegler D, Hurwitz A, Hassanein R et al. Migraine prophylaxis. A comparison of propranolol and amitriptyline. Arch Neurol 1987;44:486-9.

40 Dodick D, Freitag F, Banks J et al. Topiramate versus amitriptyline in migraine preventon: a 26-week, multicentre, randomized, double-blind, double-dummy, parallel-group noninferiority trial in adult migraineurs. Clin Ther 2009;31:542-49.

41 Mulleners W, McCrory D, Linde M. Antiepileptics in migraine prophylaxis: an updated review. Cephalalgia 2015;35:51-62.

42 Linde M, Mulleners W, Chronicle E et al. Gabapentin or pregabalin for the prophylaxis of episodic migraine in adults. Cochrane Database Syst Rev 2013 Jun 24;6:CD010609.

43 Linde M, Mulleners W, Chronicle E et al. Topiramate for the prophylaxis of episodic migraine in adults. Cochrane Database Syst Rev 2013 Jun 24;6:CD010610.

44 Linde M, Mulleners W, Chronicle E et al. Valproate (valproic acid or sodium valproate or a combination of the two) for the prophylaxis of episodic migraine in adults. Cochrane Database Syst Rev 2013 Jun 24;6:CD010611.

45 Bromley R, Weston J, Adab N et al. Treatment for epilepsy in pregnancy: neurodevelopmental outcomes in the child. Cochrane Database Syst Rev 2014 Oct 30;10:CD010236.

46 Tronvik E, Stovner L, Helde G et al. Prophylactic treatment of migraine with an angiotensin II receptor blocker: a randomized controlled trial. JAMA 2003;289:65-9.

47 Stovner L, Linde M, Gravdahl G et al. A comparative study of candesartan versus propranolol for migraine prophylaxis: A randomised, triple-blind, placebo-controlled, double cross-over study. Cephalalgia 2013;34:523-32.

48 Diener H, Matias-Guiu J, Hartung E et al. Efficacy and tolerability in migraine prophylaxis of flunarizine in reduced doses: a comparison with propranolol $160 \mathrm{mg}$ daily. Cephalalgia 2002;22:209-21.

49 Schoenen J, Jacquy J, Lenaerts M. Effectiveness of high-dose riboflavin in migraine prophylaxis. A randomized controlled trial. Neurology 1998;50:466-70.

50 Dodick D, Turkel C, DeGryse R et al. OnabotulinumtoxinA for treatment of chronic migraine: pooled results from the doubleblind, randomized, placebo-controlled phases of the PREEMPT clinical program. Headache 2010;50:921-36.

51 May A, Leone M, Afra J et al. EFNS guidelines on the treatment of cluster headache and other trigeminal-autonomic cephalalgias. Eur J Neurol 2006;13:1066-77.

52 Law S, Derry S, Moore R. Triptans for acute cluster headache. Cochrane Database Syst Rev 2013 Jul 17;7:CD008042.

53 van Vliet J, Bahra A, Martin V et al. Intranasal sumatriptan in cluster headache: randomized placebo-controlled double-blind study. Neurology 2003;60:630-3. 
54 Cohen A, Burns B, Goadsby P. High-flow oxygen for treatment of cluster headache: a randomized trial. JAMA 2009;302:2451-7.

55 Paemeleire K, Bahra A, Evers S et al. Medication-overuse headache in patients with cluster headache. Neurology 2006;67:109-13.

56 Couch J, Ziegler D. Prednisone therapy for cluster headache. Headache 1978;18:219-21.

57 Leone M, D'Amico D, Frediani F et al. Verapamil in the prophylaxis of episodic cluster headache: a double-blind study vs. placebo. Neurology 2000;54:1382-5.

58 Cohen A, Matharu M, Goadsby P. ECG abnormalities on verapamil in cluster headache. Cephalalgia 2005;25:1200.

59 Bussone G, Leone M, Peccarisi C et al. Double blind comparison of lithium and verapamil in cluster headache prophylaxis. Headache 1990;30:411-7.

60 Steiner T, Hering R, Couturier E et al. Double-blind placebo-controlled trial of lithium in episodic cluster headache. Cephalalgia 1997;17:673-5.
61 El Amrani M, Massiou H, Bousser M. A negative trial of sodium valproate in cluster headache: methodological issues. Cephalalgia 2002;22:205-8.

62 Leone M, D'Amico D, Moschiano F et al. Melatonin vs. placebo in the prophylaxis of cluster headache: a double-blind pilot study with parallel groups. Cephalalgia 1996;16:494-6.

63 Diener H. CGRP as a new target in prevention and treatment of migraine. Lancet Neurol 2014;13:1065-7.

Address for correspondence: Dr M Weatherall, Princess Margaret Migraine Clinic, Department of Neurology, Charing Cross Hospital, Fulham Palace Road, London, W6 8RF, UK. Email: mweatherall@nhs.net

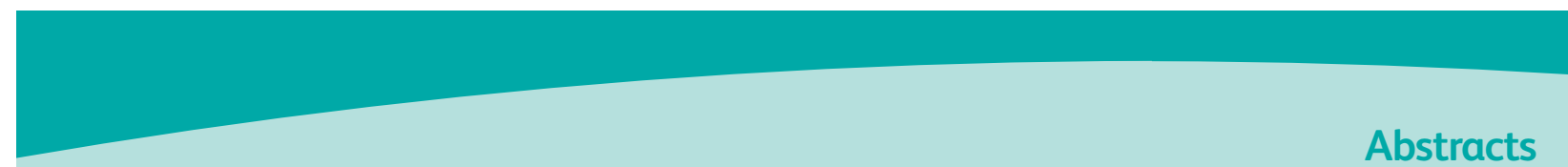

\section{Delivering the Future Hospital Medicine 2015: RCP annual conference}

The RCP is proud to announce that the abstracts presented at the Medicine 2015 annual conference are now available online.

This year's flagship conference focused on the Future Hospital Commission's vision to structure hospital services around the needs of patients, as well as implement real change across hospitals and the wider health and social care economy.

All of the abstracts published in both Clinical Medicine and Future Hospital Journal are available for free at:

http://www.clinmed.rcpjournal.org/

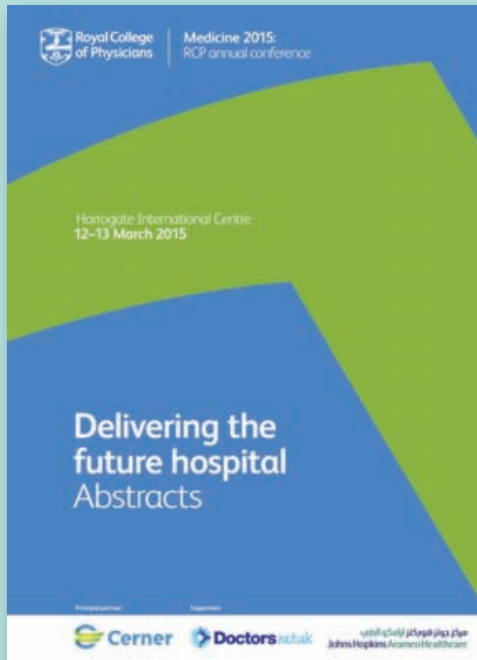

Setting higher standards 